\title{
An In Vivo Evaluation of the Effect of Repeated Admin- istration and Clearance of Targeted Contrast Agents on Molecular Imaging Signal Enhancement
}

\author{
Jason E. Streeter, Paul A. Dayton ${ }^{凶}$ \\ Joint Dept. of Biomedical Engineering, University of North Carolina-North Carolina State University, Chapel Hill, NC, USA. \\ $\square$ Corresponding author: Paul A. Dayton, Address: 304 Taylor Hall, 109 Mason Farm Road, Chapel Hill, NC, 27599-6136, Phone: (919) \\ 843-9521 Fax: (919) 843-9520, Email: padayton@bme.unc.edu. \\ (C) Ivyspring International Publisher. This is an open-access article distributed under the terms of the Creative Commons License (http://creativecommons.org/ \\ licenses/by-nc-nd/3.0/). Reproduction is permitted for personal, noncommercial use, provided that the article is in whole, unmodified, and properly cited.
}

Received: 2012.10.04; Accepted: 2012.11.21; Published: 2013.01.17

\begin{abstract}
Competitive inhibition diminishes ligand adhesion as receptor sites become occupied with competing ligands. It is unknown if this effect occurs in ultrasound molecular imaging studies where endothelial binding sites become occupied with adherent bubbles or bubble fragments. The goal of this pilot study was to assess the effect that repeated administration and clearance of targeted agents has on successive adhesion. Two groups of animals were imaged with 3-D ultrasonic molecular imaging. Injections and imaging were performed on Group I at time 0 and 60 minutes. Group 2 received injections of microbubbles at $0,15,30,45$ and 60 minutes with imaging at 0 and 60 minutes. At 60 minutes, Group I targeting relative to baseline was not significantly different from Group 2 ( $1.06 \pm 0.27$ vs. I.08 $\pm 0.34, p=0.93$ ). Data suggest that multiple injections of targeted microbubbles do not block sufficient binding sites to bias molecular imaging data in serial studies.
\end{abstract}

Key words: Microbubble, Contrast Agents, Molecular Imaging, Ultrasound, Targeted, cRGD.

\section{Introduction}

Molecular imaging with ultrasound is a modality, which is gaining interest in preclinical and clinical studies because it extends the ability to assess molecular changes to the already low-cost, non-ionizing, real-time, and widely available modality of ultrasound. The principle behind ultrasonic molecular imaging is the selective adherence of microbubble contrast agents to biomarkers expressed on the endothelium $[1,2,3]$. Once the contrast agents accumulate at the target site, they enhance the pathologic tissue via increased acoustic backscatter, thus providing indication of the presence of biomarkers associated with disease [4]. This approach enables the ability to evaluate biological changes at the molecular level (ex. angiogenic expression) before phenotypic changes occur $[4,5]$. Thus, this technique is well suited for theranostic applications such as monitoring response to therapy, which may ultimately lead to a more personalized approach to medicine $[6,7,8]$.

Often, an ultrasonic molecular imaging study requires multiple targeted microbubble injections within a single imaging study to evaluate short-term biological changes, system parameters, or novel microbubble formulations $[9,10,11,12]$. Targeted microbubbles are fitted with a ligand that allows binding to endothelial biomarkers. However, as endothelial receptors are occupied by ligands from targeted bubbles, it is probable that these receptors would no longer be available for future targeting. This might happen if either bubbles stay retained at the target site, or if fragments of the bubbles are retained at the target site after bubble destruction. Thus, if targeted 
microbubbles are injected multiple times in a single animal study, it is possible that the quantity of available binding sites is diminished over time. In the case where receptor ligands were sufficiently competitively inhibited, it would bias the results of successive molecular imaging data where multiple targeted microbubble injections are required.

We hypothesized that the amount of biomarker expression, relative to the amount of receptors occupied by ligands from targeted bubbles, would be great enough to allow for multiple targeted microbubble injections for a single animal study without discernible competitive inhibition effects. To test this hypothesis, we analyzed the in vivo molecular imaging results from two groups of animals. The first group of animals, a control group, received molecular imaging studies with microbubbles targeted to $\alpha_{v} \beta_{3}$ at time 0 and 60 minutes. The second group of animals was imaged at the same two time points, but with three additional targeted microbubble injections at time 15, 30, and 45 minutes (Figure 1). These time points were chosen to provide sufficient time for microbubbles to clear circulation prior to successive injections. Molecular imaging results were compared between groups at baseline and 60 minutes as an indication of whether or not multiple serial injections have an effect on the outcome of an ultrasonic molecular imaging experi- ment.

\section{Materials and Methods}

\section{Microbubble Preparation}

Microbubbles designed to target $\alpha_{v} \beta_{3}$ integrins were created with a 9:0.5:0.5 molar ratio of 1,2 Distearoyl- sn-Glycero-3-Phosphocholine (DSPC) (Avanti Polar Lipids, Alabaster, AL, USA), 1,2-Distearoylsn-Glycero-3-Phosphoethanolmine-N-Methoxy-Polye thylene Glycol-2000 (DSPE-PEG2000) (Avanti Polar Lipids, Alabaster, AL, USA), and DSPE-PEG2000 cross-linked to a cyclic RGD peptide (Cyclo-Arg-Ala-Asp-D-Tyr-Cys) (Peptides International, Louisville, KY, USA) as previously described [13]. The cyclic RGD peptide has previously been shown to target $\alpha_{v} \beta_{3}$-expressing vasculature, which is characteristic of angiogenic tumors [14, 15]. Competitive inhibition with free RGD shows reduction in adhesion to $\alpha_{v} \beta_{3}$ suggesting, some degree of specificity [16]. Microbubbles with a large preferentially selected mean diameter $(\sim 3.9 \mu \mathrm{m})$ have been shown to produce greater backscatter intensities in molecular imaging studies as compared to vial-shaken unsorted polydisperse distributions [13, 17]; therefore, all microbubbles in this study were size-selected via the method described by Feshitan and colleagues [18].
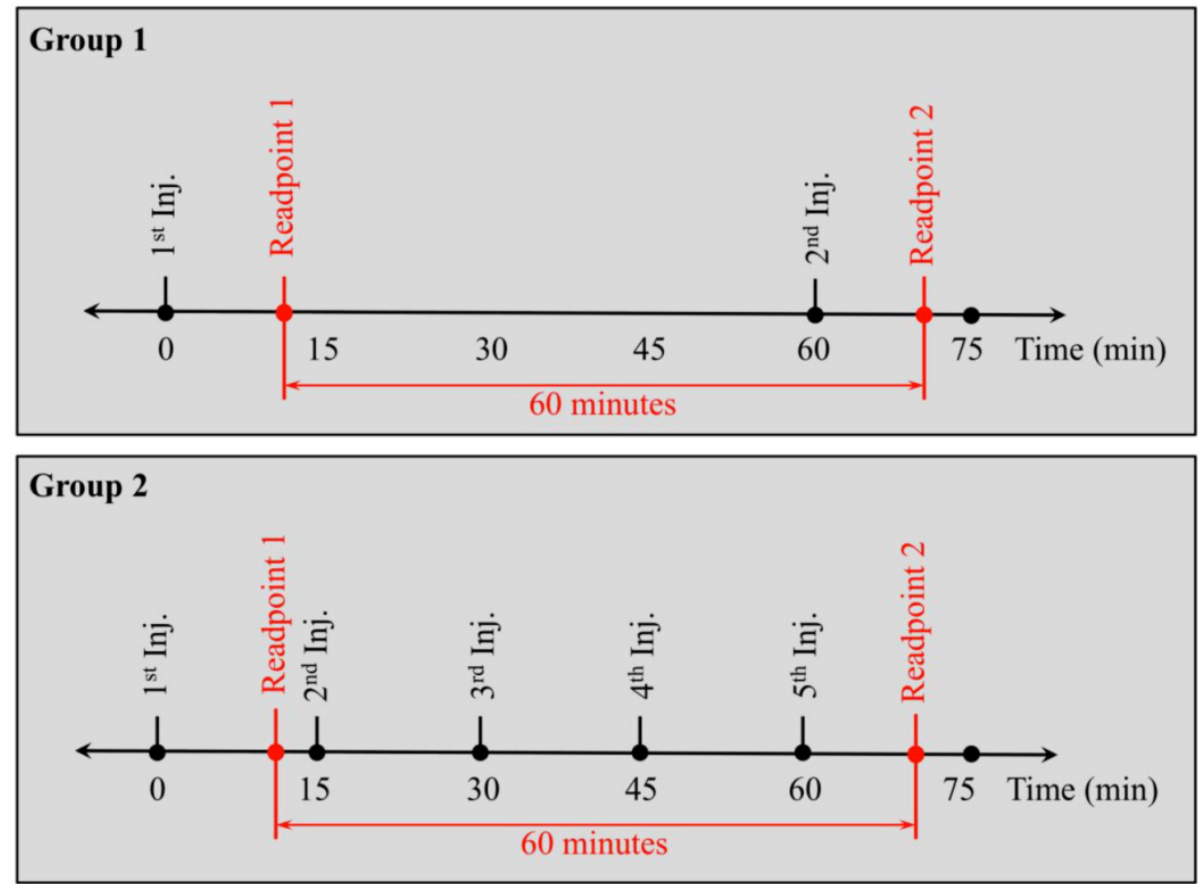

Figure I. The timelines for when bolus injections were given and the imaging readpoints for groups I and 2 . Each bolus injection, for group I and group 2, consisted of a contrast agent dose of $5 \times 10^{6}$ microbubbles. 


\section{Animals and Tumor Models}

A total of 13 Fischer 344 rats (Charles River Laboratories, Durham, NC, USA) of similar sizes $(\sim 125$ g) were used for all in vivo studies (Group $1-\mathrm{N}=7$, Group $2-\mathrm{N}=6$ ). All animal studies were conducted in accordance with the protocols approved by the University of North Carolina School of Medicine's Institutional Animal Care and Use Committee.

The tumor model used in all in vivo experiments was a rat fibrosarcoma [19]. In previous studies, this particular type of tumor has been shown to provide a good model for $\alpha_{v} \beta_{3}$ targeted molecular imaging [13, 20].

\section{Animal Preparation and contrast administra- tion}

Rodents were anesthetized for in vivo molecular imaging studies with $\sim 2 \%$ inhaled isoflurane mixed with oxygen. Once the rat was sedated, the area to be imaged was shaved with small animal hair clippers and further depilated using a chemical hair remover. A 24-gauge catheter was inserted into the tail vein of the animal for the purpose of administering microbubble contrast agents. The ultrasound transducer used in the in vivo analysis was positioned in a fixed clamp and coupled to the animal with ultrasound gel. Throughout the imaging procedure, the rodent's body temperature was maintained through the use of a temperature-controlled heating pad.

The sizes and concentrations of stock solutions for all microbubble types used in this study were measured prior to each imaging study using an Accusizer $780 \mathrm{~A}$ laser light obscuration and scattering device (Particle Sizing Systems, Santa Barbara, CA, USA). For each injection, the appropriate volume of stock solution was added to the catheter via a micropipette tip and flushed with $100 \mu \mathrm{L}$ of sterile saline such that a contrast agent dose of $5 \times 10^{6}$ microbubbles was administered consistently with each injection. Animals received less than $1.5 \mathrm{~mL}$ of total fluid volume within any 24-hour period.

\section{Clinical Imaging System}

An Acuson Sequoia 512 ultrasound imaging system (Siemens, Mountain View, CA, USA) was used to acquire all images in this study. B-mode images were collected at $14 \mathrm{MHz}$ using a 15L8 linear array transducer in spatial compounding mode as a means for selecting the regions of interest in each image plane. Microbubble contrast agents were imaged in Cadence Pulse Sequencing mode, which is nondestructive when a low MI is used [21].

To create 3-D datasets, the transducer was stepped elevationally across the tumor using a LabView (National Instruments, Austin, TX, USA) controlled motion stage (Newport, Irvine, CA, USA) as previously described [22].

\section{Molecular Imaging Protocol}

Molecular imaging data was obtained after freely circulating microbubbles had visibly cleared the animal's system (typically $\sim 10$ minutes). Once the microbubbles had cleared, one image frame of video data in Cadence Pulse Sequencing mode $(7 \mathrm{MHz}, \mathrm{MI}$ : 0.18 , dynamic range: $80 \mathrm{~dB}$ ) was collected at every 400 $\mu \mathrm{m}$ as the transducer was stepped across the tumor. Next, the imaging system was set to a microbubble-destructive setting with a center frequency of 7 $\mathrm{MHz}$ and a mechanical index of 1.9. Subsequently, the transducer was swept at $1 \mathrm{~mm} / \mathrm{sec}$ elevationally across the tumor to destroy any adherent microbubbles remaining in the vasculature. Prior studies utilizing a MI of 1.9 to destroy microbubbles suggest the absence of bioeffects at higher frequencies such as 7 $\mathrm{MHz}$ utilized in this study [23]. Finally, the system was again set to image in Cadence Pulse Sequencing mode and the tumor was re-imaged at each location across the tumor to collect background image intensity data. This baseline image set allowed us to determine the background signal for quantifying the biomarker expression. Within all imaging data sets, the gain $(-10 \mathrm{~dB})$ and transmit power (MI: 0.18) were kept constant. In a prior study, the video intensity of targeted agents retained in vivo was observed over 30 seconds using Cadence Pulse Sequencing mode at a mechanical index of 0.18 , and no loss in signal intensity was observed while imaging adherent targeted contrast agents, indicating that our imaging parameters were largely nondestructive (data not shown).

\section{Molecular Imaging Analysis}

Video data was saved from the imaging system in DICOM format and analyzed using custom MATLAB software (Mathworks, Natick, MA, USA). Volumetric intensities were obtained by summing all of the pixel intensity values for each voxel within the drawn regions of interest and dividing that value by the total number of voxels within the volume. As with previous molecular imaging studies, the intensity brightness was assumed to be correlated with the degree of $a_{v} \beta_{3}$ expression $[9,20,24,25,26]$. Final values for biomarker expression for each microbubble type were computed by subtracting the background volumetric intensity from the volumetric intensity produced by the adherent microbubbles. We used MATLAB's two-sided student's t-test to assess the statistical significance of our group. Significance be- 
tween two different distributions were considered at a value of $p<0.05$.

\section{Results}

Two different groups of animals were imaged with ultrasonic molecular imaging to determine if multiple serial injections affect the amount of adherent microbubbles detected in an individual experiment. Intensity values for each animal's readpoint were normalized to the value at the baseline measurement or time 0 . Injections and molecular imaging readpoints were performed on the control group (Group 1) at time 0 and 60 minutes after the first injection while Group 2's injections were at times 0, 15, 30,45 and 60 minutes with readpoints at 0 and 60 minutes.

Group 1 showed a $6 \%$ increase $(1.06 \pm 0.27)$ in targeting relative to baseline while Group 2 showed an $8 \%$ increase $(1.08 \pm 0.34)$ in the amount of targeted microbubbles, as determined through intensity measurements (Figure 2). Group 1 was not significantly different from Group 2 after the 60-minute readpoint $(p=0.93)$, but rather had similar targeting and similar variance. Figure 3 shows a 2 by 2 panel of a representative Group 1 tumor and a representative
Group 2 tumor at baseline and 60 minutes after. The green color represents the targeted microbubble signal detected via Cadence Pulse Sequencing mode in the experiment. The green pixels, correlated to the degree of $\alpha_{v} \beta_{3}$ expression, are overlaid onto a traditional B-mode image to illustrate the location of the targeting relative to the tumor.

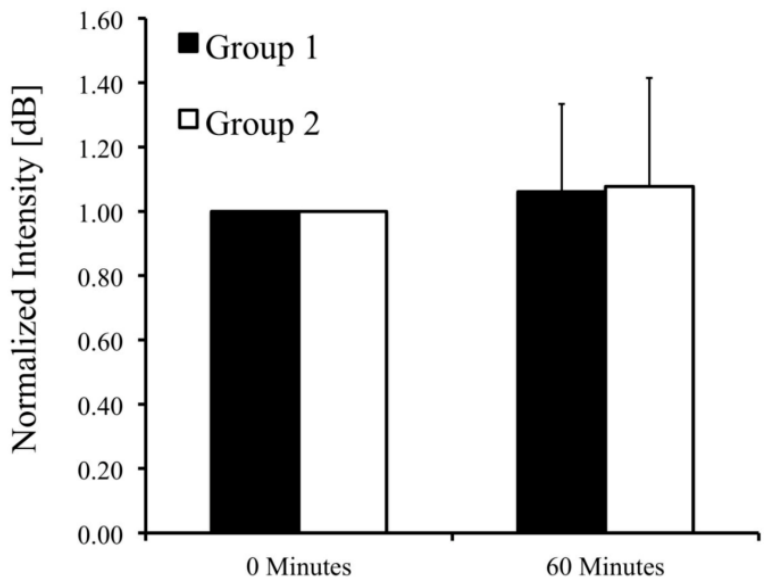

Figure 2. The percent change in volumetric targeted microbubble intensity for Groups I and 2, 60 minutes after baseline imaging. $p=0.93$ for Group I relative to Group 2 .

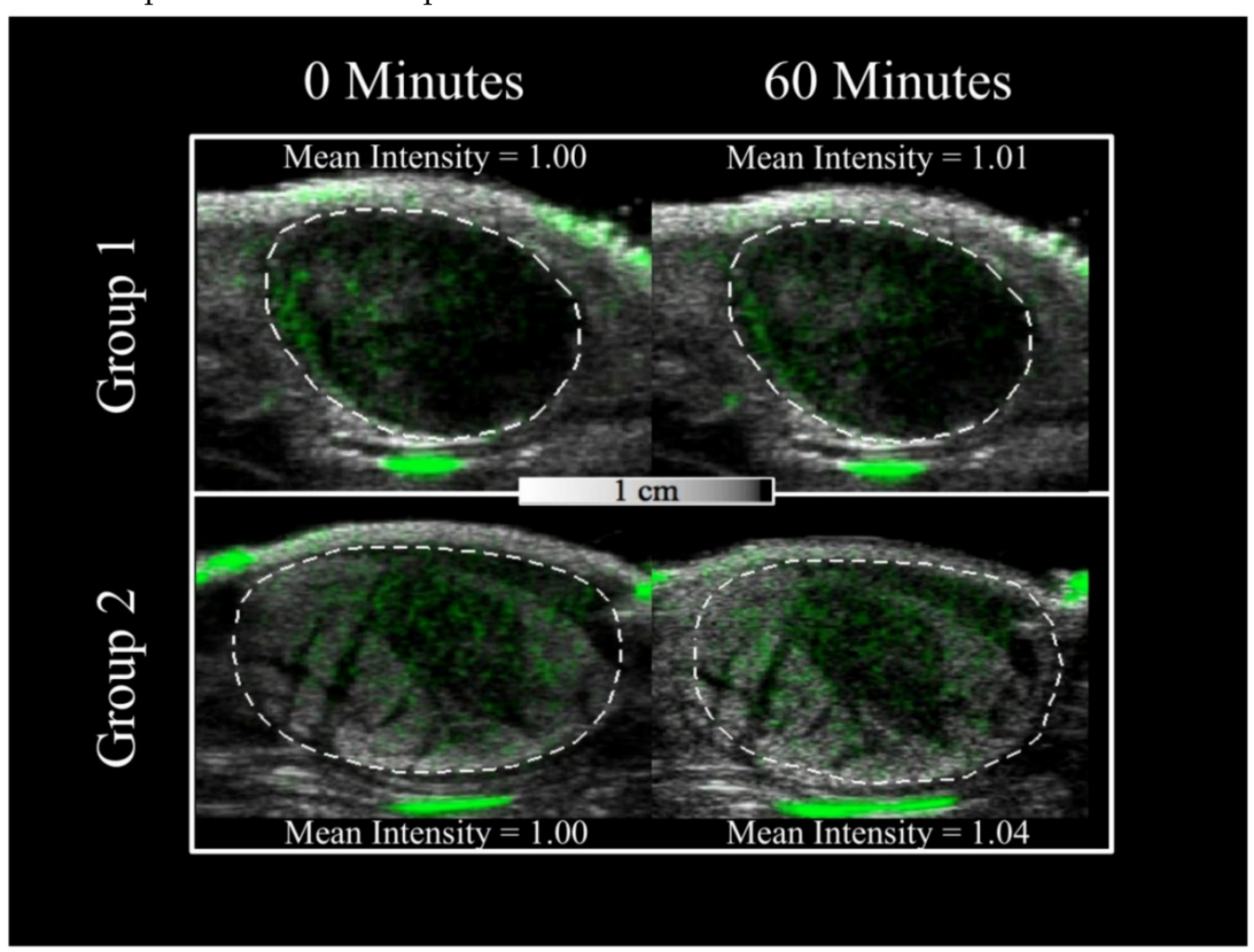

Figure 3. 2 - D ultrasound images of a representative Group I and Group 2 tumor (selected from a 3-D dataset) at baseline and 60 minutes after initial imaging. The green color is the targeted microbubble signal detected via Cadence Pulse Sequencing mode. The intensity of the green color is loosely correlated to the degree of $\alpha_{v} \beta_{3}$ expression. The green signal is overlaid onto a traditional B-mode image to illustrate the location of the targeting relative to the tumor. The white dotted line illustrates the region of interest selected for the quantification of biomarker expression. 


\section{Discussion and Conclusion}

In this study, we evaluated if successive injections of targeted microbubbles would reduce the ability of future injections of targeted bubbles to adhere to endothelial receptor ligands. While the scope of this study was limited to a short time period, as well as a specific tumor model, and a specific microbubble dose, the results are encouraging. Given the dose of cRGD-targeted microbubbles injected (which was appropriate for achieving sufficient molecular imaging signal) and the inherent variability of the molecular imaging procedure, there was no discernible statistical difference between injecting twice and injecting five times. Ultimately, this suggests that the quantity of over-expressed $\alpha_{v} \beta_{3}$ biomarkers present must be sufficiently greater than the available ligands so as not to present competitive inhibition. Thus, preliminary data suggests that serial multiple injections in a single ultrasonic molecular imaging study, do not bias or compromise endothelial retention of targeted microbubbles due to competitive inhibition from prior bound bubbles or bubble fragments. This result would be important in theranostics assays involving serial ultrasound molecular imaging exams. What our study does not address is whether or not the adhesion of targeted bubble ligands affects the tumor biology, as might be anticipated with large doses. Furthermore, we observed a slight increase in molecular targeting in both imaging models, the mechanism for which is still unknown. Future studies beyond this pilot should include larger animal numbers, different doses of contrast, different ligands, and longer time points.

\section{Acknowledgements}

This study was supported by NIH R01 EB009066 and pilot study funds from the UNC Lineberger Cancer Center. The authors would like to acknowledge the assistance of James Tsuruta in cRGD lipid conjugation that was used for targeted microbubble fabrication, as well as Charlene Ross and the Animal Studies Core Facility for their technical assistance in tumor implantation.

\section{Competing Interests}

The authors have declared that no competing interest exists.

\section{References}

1. Gessner RC, Dayton PA. Advances in molecular imaging with ultrasound. Mol Imaging .2010 Jun;9(3):117-27.

2. Kiessling F, Fokong S, Koczera P, Lederle W, Lammers T. Ultrasound microbubbles for molecular diagnosis, therapy, and theranostics. J Nucl Med. 2012 Mar;53(3):345-8.
3. Weller GER, Villanueva FS, Tom EM, Wagner WR. Targeted ultrasound contrast agents: in vitro assessment of endothelial dysfunction and multi-targeting to ICAM-1 and sialyl Lewisx. Biotechnol Bioeng. 2005 Dec;92(6):780-8.

4. Dayton PA, Ferrara KW. Targeted imaging using ultrasound. J Magn Reson Imaging. 2002 Oct;16(4):362-77.

5. Leong-Poi H, Christiansen JP, Klibanov AL, Kaul S, Lindner JR. Noninvasive assessment of angiogenesis by ultrasound and microbubbles targeted to alpha(v)-integrins. Circulation. 2003 Jan;107(3):455-60.

6. Streeter JE, Herrera-Loeza SG, Neel NF, Yeh JJ, Dayton PA. A Comparative Evaluation of Ultrasound Molecular imaging, Perfusion Imaging, and Volume Measurements in Evaluating Response to Therapy in Patient-Derived Xenografts. Technol Cancer Res Treat. 2012; In Press.

7. Sorace AG, Saini R, Mahoney M, Hoyt K. Molecular ultrasound imaging using a targeted contrast agent for assessing early tumor response to antiangiogenic therapy. J Ultrasound Med. 2012 Oct;31(10):1543-50.

8. Sirsi SR, Flexman ML, Vlachos F, Huang J, Hernandez SL, Kim HK, Johung TB, Gander JW, Reichstein AR, Lampl BS, Wang A, Hielscher AH, Kandel JJ, Yamashiro DJ, Borden MA. Contrast ultrasound imaging for identification of early responder tumor models to anti-angiogenic therapy. Ultrasound Med Biol. 2012 Jun;38(6):1019-29.

9. Gessner RC, Streeter JE, Kothadia R, Feingold S, Dayton PA. An In Vivo Validation of the Application of Acoustic Radiation Force to Enhance the Diagnostic Utility of Molecular Imaging Using 3-D Ultrasound. Ultrasound Med Biol. 2012 Apr;38(4):651-60.

10. Borden MA, Streeter JE, Sirsi SR, Dayton PA. An acoustically targeted, immune-stealth system for in vivo ultrasound assessment of angiogenesis. Manuscript in preparation.

11. Frinking PJA, Tardy I, Théraulaz M, Arditi M, Powers J, Pochon S, Tranquart F. Effects of Acoustic Radiation Force on the Binding Efficiency of BR55, a VEGFR2-Specific Ultrasound Contrast Agent. Ultrasound Med Biol. 2012 Aug;38(8):1460-9.

12. Willmann JK, Lutz AM, Paulmurugan R, Patel MR, Chu P, Rosenberg J, Gambhir SS. Dual-targeted contrast agent for US assessment of tumor angiogenesis in vivo. Radiology. 2008 Sep;248(3):936-44.

13. Streeter JE, Gessner RC, Miles I, Dayton PA. Improving sensitivity in ultrasound molecular imaging by tailoring contrast agent size distribution: in vivo studies. Mol Imaging. 2010 Apr;9(2):87-95.

14. Ruoslahti E. Integrins as signaling molecules and targets for tumor therapy. Kidney Int. 1997 May;51(5):1413-7.

15. Haubner R, Wester H-J. Radiolabeled tracers for imaging of tumor angiogenesis and evaluation of anti-angiogenic therapies. Curr Pharm Des. 2004;10(13):1439-55.

16. Dayton PA, Pearson D, Clark J, Simon S, Schumann PA, Zutshi R, Matsunaga TO, Ferrara KW. Ultrasonic analysis of peptide- and antibody-targeted microbubble contrast agents for molecular imaging of alphavbeta3-expressing cells. Mol Imaging. 2004 Apr;3(2):125-34.

17. Sirsi S, Feshitan JA, Kwan J, Homma S, Borden MA. Effect of microbubble size on fundamental mode high frequency ultrasound imaging in mice. Ultrasound Med Biol. 2010 Jun;36(6):935-48.

18. Feshitan JA, Chen CC, Kwan JJ, Borden MA. Microbubble size isolation by differential centrifugation. J Colloid Interface Sci. 2009 Jan 15;329(2):316-24.

19. Schroeder T, Yuan H, Viglianti BL, Peltz C, Asopa S, Vujaskovic Z, Dewhirst MW. Spatial heterogeneity and oxygen dependence of glucose consumption in R3230Ac and fibrosarcomas of the Fischer 344 rat. Cancer Res. 2005 Jun;65(12):5163-71.

20. Streeter JE, Gessner RC, Tsuruta J, Feingold S, Dayton PA. Assessment of Molecular Imaging of Angiogenesis with Three-Dimensional Ultrasonography. Mol Imaging. 2011 Dec;10(6):460-68.

21. Stieger SM, Dayton PA, Borden MA, Caskey CF, Griffey SM, Wisner ER, Ferrara KW. Imaging of angiogenesis using Cadence contrast pulse sequencing and targeted contrast agents. Contrast Media Mol Imaging. Jan-Feb 2008;3(1):9-18.

22. Feingold S, Gessner RC, Guracar IM, Dayton PA. Quantitative volumetric perfusion mapping of the microvasculature using contrast ultrasound. Invest Radiol. 2010 Oct;45(10):669-74.

23. Johnson KA, Cianciolo R, Gessner RC, Dayton PA. A pilot study to assess markers of renal damage in the rodent kidney after exposure to 7 $\mathrm{MHz}$ ultrasound pulse sequences designed to cause microbubble translation and disruption. Ultrasound Med Biol. 2012 Jan;38(1):168-72.

24. Ellegala DB, Leong-Poi H, Carpenter JE, Klibanov AL, Kaul S, Shaffrey ME, Sklenar J, Lindner JR. Imaging tumor angiogenesis with contrast ultrasound and microbubbles targeted to alpha(v)beta3. Circulation. 2003 Jul;108(3):336-41. 
25. Warram JM, Sorace AG, Saini R, Umphrey HR, Zinn KR, Hoyt K. A triple-targeted ultrasound contrast agent provides improved localization to tumor vasculature. J Ultrasound Med. 2011 Jul;30(7):921-31.

26. Tardy I, Pochon S, Theraulaz M, Emmel P, Passantino L, Tranquart F, Schneider M. Ultrasound molecular imaging of VEGFR2 in a rat prostate tumor model using BR55. Invest Radiol. 2010 Oct;45(10):573-8. 